\title{
Possible origin of viscosity in the Keplerian accretion disks due to secondary perturbation: Turbulent transport without magnetic field
}

\author{
Banibrata Mukhopadhyay and Kanak Saha \\ Astronomy and Astrophysics Program, Department of Physics, Indian Institute of Science, Bangalore \\ 560012, India; bm@physics.iisc.ernet.in
}

\begin{abstract}
The origin of hydrodynamic turbulence in rotating shear flow is a long standing puzzle. Resolving it is especially important in astrophysics when the flow angular momentum profile is Keplerian which forms an accretion disk having negligible molecular viscosity. Hence, any viscosity in such systems must be due to turbulence, arguably governed by magnetorotational instability especially when temperature $T \gtrsim 10^{5}$. However, such disks around quiescent cataclysmic variables, protoplanetary and star-forming disks, the outer regions of disks in active galactic nuclei are practically neutral in charge because of their low temperature, and thus expected not to be coupled with the magnetic field appropriately to generate any transport due to the magnetorotational instability. This flow is similar to plane Couette flow including the Coriolis force, at least locally. What drives their turbulence and then transport, when such flows do not exhibit any unstable mode under linear hydrodynamic perturbation? We demonstrate that the threedimensional secondary disturbance to the primarily perturbed flow triggering elliptical instability may generate significant turbulent viscosity ranging $0.0001 \lesssim \nu_{t} \lesssim 0.1$ to explain transport in accretion flows.
\end{abstract}

Key words: accretion, accretion disks — hydrodynamics — turbulence — instabilities

\section{INTRODUCTION}

One of the main problems behind the origin of hydrodynamic turbulence in shear flow is that there is a significant mismatch between the predictions of linear theory and experimental data. For example, in the case of plane Couette flow, laboratory experiments and numerical simulations show that the flow may be turbulent at a Reynolds number as low as $R e \sim 350$, while according to the linear theory the flow should be stable for all Re. Similar mismatch between theoretical results and observations is found in astrophysical contexts, where the accretion flow of neutral gas with Keplerian angular momentum profile, which essentially behaves like rotating shear flow, is a common subject. Examples of such flow systems are accretion disks around quiescent cataclysmic variables (Gammie \& Menou 1998), protoplanetary and star-forming disks (Blaes \& Balbus 1994), and the outer regions of disks in active galactic nuclei (Menou \& Quataert 2001).

A Keplerian accretion disk flow having a very low molecular viscosity must generate turbulence and successively diffusive viscosity, which support the transfer of mass inwards and angular momentum outwards. However, theoretically this flow, in absence of magnetic field, never exhibits any unstable mode which could trigger turbulence in the system. On the other hand, the laboratory experiments of TaylorCouette systems, which are similar to Keplerian disks, seem to indicate that although the Coriolis force delays the onset of turbulence, the flow is ultimately unstable to turbulence for Reynolds numbers larger than a few thousand (Richard \& Zahn 2001), even for subcritical systems. Indeed, Bech \& Anderson (1997) see turbulence persisting in numerical simulations of subcritical rotating flows for large enough Reynolds numbers.

How does shearing flow that is linearly stable to perturbations switch to a turbulent state? Since last decade, many authors including ourselves have come forward with a possible explanation of this fact 
based on bypass transition (see, Butler \& Farrell 1992, Reddy \& Henningson 1993. Trefethen et al. 1993, Chagelishvili et al. 2003. Umurhan \& Regev 2004, Mukhopadhyay et al. 2005 and references therein) where the decaying linear modes show an arbitrarily large transient energy growth at a suitably tuned perturbation. In lieu of linear instabilities e.g. magnetorotational instability, the transient energy growth, supplemented by a non-linear feedback process to repopulate the growing disturbance, could plausibly sustain turbulence for large enough Reynolds numbers.

The behavior of shear flows, however, in the presence of rotation is enormously different compared to that in absence of rotation. The Coriolis effect is the main culprit behind this change in behavior killing any growth of energy even of transient kind in the presence of rotation. In the case of shear flow with a varying angular velocity profile, e.g. Keplerian accretion flow, the above mentioned transient energy growth is insignificant for threedimensional perturbations. To overcome this limitation, it is necessary to invoke additional effects. Various kinds of secondary instability, such as the elliptical instability, are widely discussed as a possible route to self-sustained turbulence in linearly perturbed shear flows (see, e.g. Pierrehumbert 1986, Bayly 1986, Craik \& Criminale 1986, Landman \& Saffman 1987, Hellberg \& Orszag 1988, Waleffe 1989, Craik 1989, Le Diześ et al. 1996, Kerswell 2002). These effects, which generate threedimensional instabilities of a twodimensional flow with elliptical streamlines, have been proposed as generic mechanism for the breakdown of many twodimensional high Reynolds number flows whose vortex structures can be locally seen as elliptical streamlines. Recently, one of the present authors has studied the secondary perturbation and corresponding elliptical vortex effects in accretion disks and pinpointed that they can be the seed of threedimensional hydrodynamic instability (Mukhopadhyay 2006). Subsequently, by numerical simulation, this has been shown to be one of the possible sources to generate turbulence to form large objects from the dusty gas surrounding a young star (Cuzzi 2007, Ormel et al. 2008). Moreover, vortex generation in the unmagnetized protoplanetary disks has been furnished by hydrodynamic turbulence (de Val-Borro et al. 2007) which leads to planet formation, and angular momentum transport in disks. However, whether they lead to non-linear feedback and threedimensional turbulence are yet to be shown explicitly.

Here we plan to show in detail that threedimensional secondary perturbation generating large growth in the flow time scale may generate significant turbulent viscosity in rotating shear flows, more precisely in plane shear flows with the Coriolis force. The plane shear flow with the Coriolis force essentially behaves as a local patch of a rotating shear flow. Possibility of significant turbulent transport in such flows by threedimensional perturbation opens a new window to explain accretion process in flows which are neutral in charge. In particular, we address the issue of deriving turbulent viscosity and the Shakura-Sunyaev viscosity parameter $\alpha$ (Shakura \& Syunyaev 1973) from a pure hydrodynamical perspective 1 . This is important for understanding accretion flows in cold charge neutral medium.

It is important to note that transition to turbulence is not a unique process, but it depends on the initial condition/disturbance and the nature of the flow (Schmid \& Henningson 2001, Criminale et al. 2003). In fact, it is known that even in the presence of secondary instability, linearly unstable base flows may reach to a non-turbulent saturated state. However, turbulence definitely belongs to the nonlinear regime and it is exhibited only in the situations when large growth of perturbation switches the system over the non-linear regime. As our present goal is to understand the possible origin of hydrodynamic turbulence, we consider those situations when large energy growth governs non-linearity.

The paper is organized as follows. In the next section, we first recall the perturbation established previously (Mukhopadhyay 2006) due to secondary disturbance in the Keplerian flow and then discuss the range of corresponding Reynolds number and the solutions. Subsequently, we estimate the corresponding turbulent viscosity of hydrodynamic origin in $\S 3$. We end in $\S 4$ by discussing implications of our results.

\section{PERTURBATION AND RANGE OF REYNOLDS NUMBER}

Considering a twodimensional velocity perturbation $\boldsymbol{w}=\left(w_{x}(x, y, z, t), w_{y}(x, y, z, t), 0\right)$, and pressure perturbation $p_{p}(x, y, z, t)$ in a small section of the Keplerian shear flow/disk, the linearized Navier-Stokes and continuity equations for the incompressible fluid with plane background shear in the presence of a

${ }^{1}$ A preliminary calculation of such $\alpha$ has been appeared in a collected volume of Gravity Research Foundation (Mukhopadhyay 2008). 
Coriolis component can be written in dimensionless units as (see Mukhopadhyay et al. 2005 for a detailed description)

$$
\begin{gathered}
\frac{d w_{x}}{d t}=2 \Omega w_{y}-\frac{\partial p_{p}}{\partial x}+\frac{1}{R e} \nabla^{2} w_{x} \\
\frac{d w_{y}}{d t}=\Omega(q-2) w_{x}-\frac{\partial p_{p}}{\partial y}+\frac{1}{R e} \nabla^{2} w_{y} \\
\frac{\partial w_{x}}{\partial x}+\frac{\partial w_{y}}{\partial y}=0 .
\end{gathered}
$$

We consider the standard no-slip boundary condition such that $w_{x}=w_{y}=0$ at $x= \pm 1$ and according to the choice of variables in the coordinate system $\Omega=1 / q$. Here $(x, y, z)$ is a local Cartesian coordinate system centered at a point $(r, \phi)$ in the disk (Mukhopadhyay et al. 2005) such that $d r=x$ and $r d \phi=y$.

When the Reynolds number is very large, the solution of eqns. (1), (2) and (3) are given by (Mukhopadhyay et al. 2005)

$$
w_{x}=\zeta \frac{k_{y}}{l^{2}} \sin \left(k_{x} x+k_{y} y\right), w_{y}=-\zeta \frac{k_{x}}{l^{2}} \sin \left(k_{x} x+k_{y} y\right)
$$

where $\zeta$ is the amplitude of vorticity perturbation, $k_{x}$ and $k_{y}$ are the components of primary perturbation wavevector and $l=\sqrt{k_{x}^{2}+k_{y}^{2}}$. Under this primary perturbation, the flow velocity and pressure modify to

$$
\boldsymbol{U}=\boldsymbol{U}^{p}+\boldsymbol{w}=\left(w_{x},-x+w_{y}, 0\right)=\mathbf{A} \cdot \boldsymbol{d}, \quad \bar{P}=\bar{p}+p_{p},
$$

where $\boldsymbol{U}^{p}, \bar{p}$ are background velocity and pressure respectively, $\mathbf{A}$ is a tensor of rank 2 . Here $k_{x}=k_{x 0}+k_{y} t$, which basically is the radial component of primary perturbation wavevector, varying from $-\infty$ to a small number, where $k_{x 0}$ is a large negative number: $\left|k_{x 0}\right| \sim R e^{1 / 3} \sim t_{\max }$ (Mukhopadhyay et al. 2005).

Now we concentrate on a further small patch of the primarily perturbed flow such that the spatial scale is very small compared to the wavelength of primary perturbation satisfying $\sin \left(k_{x} x+k_{y} y\right) \sim k_{x} x=f \lesssim 1$. In fact, $f \sim 1$ at close to the boundary of the patch when $y \rightarrow 0$ and $2 \pi / k_{y}$, and at an intermediate location $f \ll 1$. As $\left|k_{x}\right|$ varies from a large number to close to unity, the size of the primary perturbation box in the $x$-direction is $1 / k_{x} \lesssim 1$ when $k_{y} \sim 1$, fixed. Hence, this further small patch must be confined to a region: $-a \lesssim x \lesssim a$, when $f /\left|k_{x 0}\right| \lesssim a \lesssim f$. Clearly, in this patch, $\boldsymbol{U}$ in eqn. (5) describes a flow having generalized elliptical streamlines with $\epsilon=\left(k_{x} / l\right)^{2}$, a parameter related to the measure of eccentricity 2 , running from 0 to 1 as the perturbation evolves. It was already shown (Mukhopadhyay 2006) that a secondary perturbation in this background may grow exponentially leading the flow unstable. We use this unstable flow in $\S 3$, which was extensively discussed earlier (Mukhopadhyay 2006), to derive $\nu_{t}$ and $\alpha$.

As we focus on the secondary perturbation at a small patch of the primarily perturbed shearing box, the variation of primary perturbation appears insignificant in the patch compared to that of the secondary one. Depending on the primary perturbation wavevector at a particular instant, the size of the secondary patch is appropriately adjusted. In fact $\epsilon$ varies very very slowly and marginally deviates from unity in the time interval when $k_{x}$ varies from $k_{x 0}$ (large negative) to, say, -10 . Even when $k_{x}$ tends to $-3, \epsilon$ changes to $\sim 0.9$ only. Therefore, $\epsilon$ and thus A practically remains constant.

\subsection{Range of Reynolds number}

Due to consecutive choice of small boxes/patches, the Reynolds number in the secondary flow is restricted with a particular choice of that in the primary flow. Here in the interest of clarity, we work with the original dimensioned units. The Reynolds number at the primary box is defined as

$$
R e_{p}=\frac{U_{0} L}{\nu}=\frac{q \Omega_{0} L^{2}}{\nu},
$$

\footnotetext{
${ }^{2}$ Note that $\epsilon$ is a parameter related to the measure of eccentricity but not the eccentricity itself.
} 
where $2 L$ is the box size in the $x$-direction and $2 U_{0}$ is the relative velocity of the fluid elements in the box between two walls along the $y$-direction. Now we recall the secondary perturbation at a smaller patch, extended from $-L_{s}$ to $+L_{s}$, such that $\left|L_{s}\right| \sim a L$. To meet our requirement $\sin \left(k_{x} x+k_{y} y\right) \sim k_{x} x+k_{y} y$, we remind that the small patch size needs to be adjusted. Therefore, the Reynolds number at the secondary box is given by

$$
R e_{s}=\frac{q \Omega_{0} L_{s}^{2}}{\nu} \sim \frac{q \Omega_{0} a^{2} L^{2}}{\nu}
$$

Hence,

$$
\frac{R e_{p}}{R e_{s}} \sim \frac{1}{a^{2}} \sim \frac{k_{x}^{2}}{f^{2}}
$$

At the beginning of the primary perturbation $k_{x}=k_{x 0}$ and thus $\epsilon=1$. At this stage, the secondary box size $L_{s}=L f / k_{x 0}$ and $R e_{p} \gtrsim k_{x 0}^{2} R e_{s}$. With time $k_{x}$ decreases in magnitude but $\epsilon$ deviates little from unity until $k_{x} \sim-3$ when $\epsilon=0.9$. Hence $\mathbf{A}$ can be considered constant approximately as described above. At this stage $R e_{p} \geq 9 R e_{s}$, atleast an order of magnitude higher than $R e_{s}$. If the energy growth due to primary perturbation is maximized for $k_{x}=k_{x, \min }=\pi$ (Mukhopadhyay et al. 2005), then the range of $R e$ for the secondary perturbation is given by $R e_{p} f^{2} / k_{x 0}^{2} \lesssim R e_{s} \lesssim R e_{p} f^{2} / 10$. At $k_{x}=\pi, R e_{s}$ is atleast an order of magnitude lower than $R e_{p}$. When $k_{x, \text { mim }}=1, R e_{p} \sim R e_{s}$ for $f \sim 1$. In general $R e_{p} f^{2} / k_{x 0}^{2} \lesssim R e_{s} \lesssim R e_{p} f^{2} / k_{x, \min }^{2}$.

\subsection{Solution}

Following previous work (Mukhopadhyay 2006), the general solution for the evolution of secondary perturbation in the flow discussed above can be written in terms of Floquet modes

$$
u_{i}(t)=\exp (\sigma t) f_{i}(\phi) \exp \left[i\left(k_{1} x+k_{2} y+k_{3} z\right)\right],
$$

where $\phi=\varpi t, f_{i}(\phi)$ is a periodic function having time-period $T=2 \pi / \varpi, \sigma$ is the Floquet exponent, $k_{1}, k_{2}, k_{3}$ are the components of wavevector of the secondary perturbation. Note that $\sigma$ is different at different $\epsilon$. Clearly, if $\sigma$ is positive, then the system is unstable. The detailed solutions were discussed elsewhere (Mukhopadhyay 2006) what we will not repeat here.

In principle, $k_{x}$ varies with time and thus $\mathbf{A}$ does so. Thus, generalizing the solution (9) for a (slowly) varying $\mathbf{A}$, we obtain

$$
u_{i}(t)=\exp \left(\int \sigma(t) d t\right) f_{i}(\phi) \exp \left[i\left(k_{1} x+k_{2} y+k_{3} z\right)\right],
$$

where $\phi=\int \varpi(t) d t$. The eqns. (9) and (10) practically describe the solutions for the entire parameter regime exhibiting elliptical vortices which are very favorable for the elliptical instability to trigger.

For the present purpose, the physically interesting quantity is the energy growth of perturbation which is given by

$$
G=\frac{\left|u_{i}(t)\right|^{2}}{\left|u_{i}(0)\right|^{2}}=\exp [2 \Sigma(t)] \frac{f_{i}^{2}(\phi)}{f_{i}^{2}(0)}
$$

where $\Sigma(t)=\int \sigma(t) d t$ and $t=\left(k_{x}-k_{x 0}\right) / k_{y}$. As $k_{x}(t)$ varies from a large negative value, $k_{x 0}$, to 0 , $t$ increases from 0 to $t_{\max }=-k_{x 0} / k_{y}$. Thus, the energy growth is controlled by the quantity $\Sigma(t)$, as $f_{i}^{2}(\phi) / f_{i}^{2}(0)$ simply appears to be a phase factor. Therefore, our aim should be to evaluate $\Sigma$ for various possible perturbations.

Let us specifically concentrate on the Keplerian accretion flows. Figure 1 shows the variation of maximum velocity growth rate, $\sigma_{\max }$, as a function of eccentricity parameter, $\epsilon$, for the various choices of amplitude of vorticity, $\zeta$. By "maximum" we refer the quantity obtained by maximizing over the vertical component of the wavevector, $k_{3}$. At large $\epsilon$ (as well as large $k_{x}$ ), when $\zeta$ is large, the background flow structure, $\mathbf{A}$, is elliptical with high eccentricity. Therefore a vertical perturbation triggers the best growing 

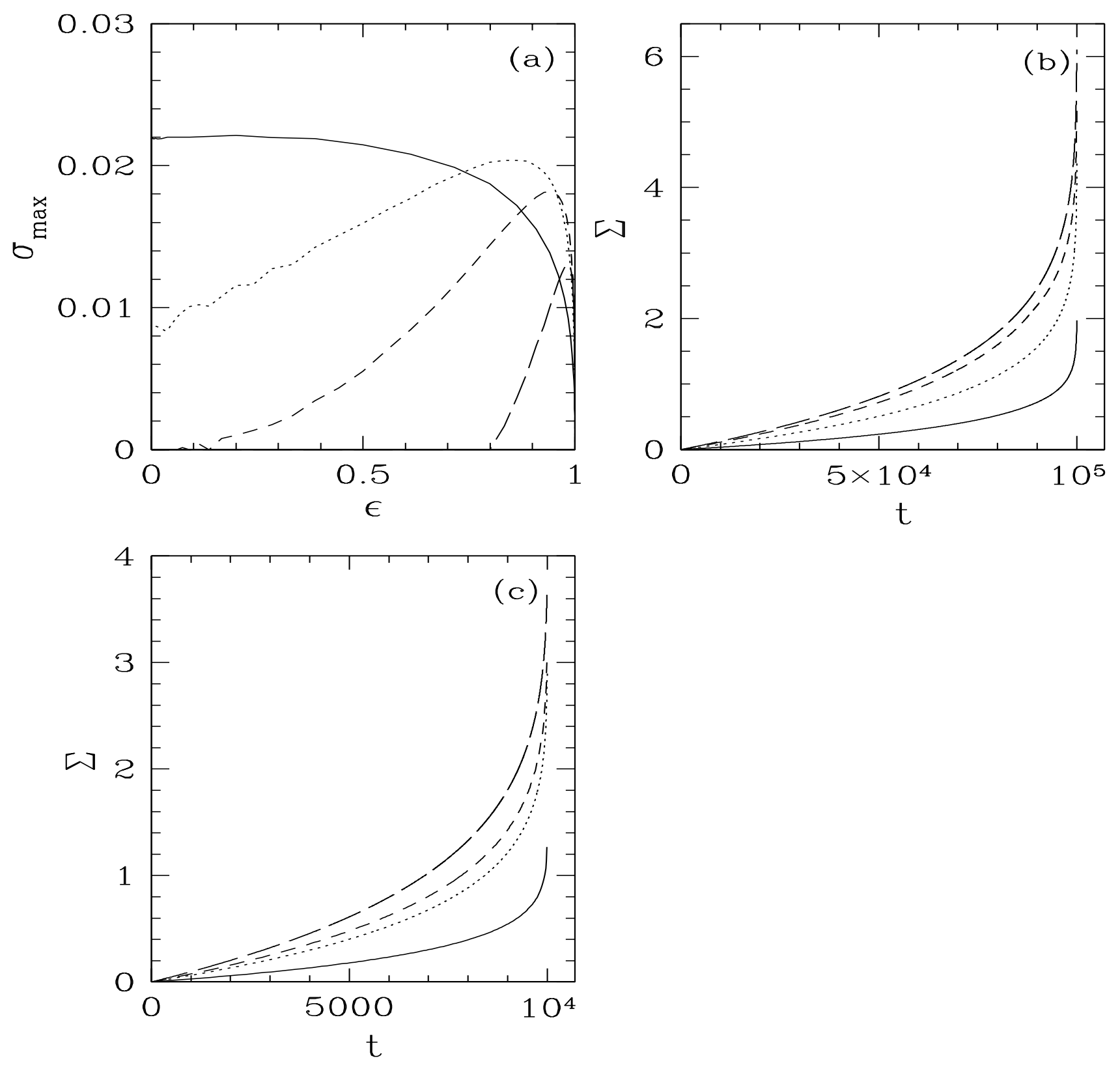

Fig. 1 (a) Variation of maximum velocity growth rate as a function of eccentricity parameter. Solid, dotted, dashed and long-dashed curves indicate the results for $\zeta=0.01,0.05,0.1,0.2$ respectively (Mukhopadhyay 2006). (b) Variation of $\Sigma$ as a function of time for $k_{x 0}=-10^{5}$, when various curves are same as of (a). (c) Same as (b) but for $k_{x 0}=-10^{4}$. Other parameters are $k_{y 0}=1, k_{10}=0,\left|\boldsymbol{k}_{0}\right|=1$, and $q=3 / 2$. 
mode into the system. However, with the decrease of $\zeta, \mathbf{A}$ approaches to that of the plane shear and thus the growth rate decreases significantly. At this stage, the corresponding best perturbation is threedimensional but not the vertical one.

At small $\epsilon$ (and then small $k_{x}$ ), when $\zeta$ is large the eccentricity of the background elliptical flow decreases significantly, and thus the growth rate decreases. In this low eccentric flow, the best growth rate arises due to the twodimensional perturbation. On the other hand, when $\zeta$ is small, the background reduces to that of the plane shear flow. Therefore, the growth rate increases according to the shearing effects, as described by Mukhopadhyay et al. 2005. An interesting fact to note is that except the case of small $\epsilon\left(k_{x}\right)$ with a large $\zeta$, the growth rate maximizes for the threedimensional perturbation. Moreover, at a large $\zeta$ and a large $\epsilon$, the best growth rate arises due to a vertical (or almost vertical) perturbation.

As the accretion time scale is an important factor, for the present purpose, physically interesting quantity is $\Sigma$ rather than $\sigma$ itself. Figures $1 \mathrm{~b}$,c show the variation of $\Sigma$ as a function of $t$ at various $\zeta$. As the perturbation evolves with time, the corresponding $\Sigma$ increases. It is also clear that $\Sigma$ and then corresponding growth increases with the increase of $\left|k_{x 0}\right|$ (and then $R e$ ), i.e. the increase of accretion time scale, in addition to the increase of $\zeta$. In Table 1, we enlist the approximate values of maximum growth factor, as follows from eqn. (11), corresponding to $\Sigma_{\max }=\int_{0}^{t_{\max }} \sigma d t$, for the cases shown in Figs. 11b,c. When $k_{x 0}=-10^{4}$, $R e_{p} \sim 10^{12}$ (as $R e_{p} \sim t_{\max }^{3} \sim k_{x 0}^{3}$ ) and from eqn. (8) $R e_{s}(f=1) \gtrsim 10^{4}$, the maximum growth factor is significant for a large amplitude of vorticity perturbation i.e. $\zeta>0$.1. However, the growth factor increases with the increase of $\operatorname{Re}_{p}$ and when $\operatorname{Re}_{p} \sim 10^{15}$, and then $\operatorname{Re}_{s}(f=1) \gtrsim 10^{5}$, it is quite significant for an amplitude of vorticity perturbations as small as 0.05 . Therefore, it appears that a suitable threedimensional secondary perturbation efficiently triggers elliptical instability and possible turbulence in rotating shear flows including accretion disks.

Table 1

Maximum energy growth corresponding to cases shown in Figs.1b,c

\begin{tabular}{cccc}
\hline \hline$\left|k_{x 0}\right|$ & $\zeta$ & $\Sigma_{\max }$ & $G_{\max }$ \\
\hline \hline $10^{5}$ & 0.2 & 6.1 & $2 \times 10^{5}$ \\
$10^{5}$ & 0.1 & 5.2 & $3.3 \times 10^{4}$ \\
$10^{5}$ & 0.05 & 4.43 & $7 \times 10^{3}$ \\
$10^{5}$ & 0.01 & 1.97 & 52 \\
\hline \hline $10^{4}$ & 0.2 & 3.65 & 1500 \\
$10^{4}$ & 0.1 & 3 & 400 \\
$10^{4}$ & 0.05 & 2.9 & 330 \\
$10^{4}$ & 0.01 & 1.27 & 13 \\
\hline \hline
\end{tabular}

\section{TURBULENT VISCOSITY}

Here we attempt to quantify the turbulence by parametrizing it in terms of the viscosity. This is essentially important, as explained in $\S 1$, in flows like astrophysical accretion disks, where molecular viscosity is negligible, to explain any transport therein.

The tangential stress at a point $(r, \phi)$ of a rotating flow exhibiting turbulence is

$$
W_{r \phi}=\nu_{t} r \frac{d \Omega}{d r}=-\nu_{t} q \Omega,
$$

where $\nu_{t}$ is the turbulent viscosity and $\Omega=\Omega_{0}\left(r / r_{0}\right)^{-q}$. Note that $q=3 / 2$ for the Keplerian angular velocity profile. The perturbation described above is expected to govern the nonlinearity after certain time, say $t_{g}$. We also assume that the nonlinearity leads to turbulence attributing the fact that at the initiation of turbulence the eddy velocity is same as the perturbation velocity. Therefore, we obtain the averaged tangential stress due to perturbation at $t=t_{g}$

$$
\begin{array}{r}
T_{r \phi}\left(t_{g}\right) \rightarrow T_{x y}\left(t_{g}\right)=<u_{x} u_{y}> \\
=\frac{k_{2}}{4 \pi L_{s}} \int_{-L_{s}}^{+L_{s}} \int_{0}^{2 \pi / k_{2}} u_{x}\left(t_{g}\right) u_{y}\left(t_{g}\right) d x d y
\end{array}
$$


where we remind that the azimuthal flow is considered to be periodic in $y=2 \pi / k_{2}$.

Now combining eqns. (12), (13) and after some algebra we obtain

$$
\bar{\nu}_{t}=-\frac{T_{x y}}{q \Omega\left(\frac{h}{r}\right) M}
$$

where $T_{x y}=\int W_{x y} d x d y, M=\Omega x / c_{s}$ and $\bar{\nu}_{t}$ denotes the averaged $\nu_{t}$ in the small section, computed here at $t=t_{g}$.

Without any proper knowledge of turbulence in Keplerian flows which arise in accretion disks, Shakura $\&$ Sunyaev (Shakura \& Syunyaev 1973) parametrized it by a constant $\alpha$ considering $W_{r \phi}$ to be proportional to the sound speed, $c_{s}$, given by

$$
W_{r \phi}=-\alpha c_{s}^{2}
$$

$\alpha$ is called the Shakura-Sunyaev viscosity parameter. They assumed that the small section under consideration to be isotropic so that scaled the characteristic length $l_{t}$ of turbulence in terms of the largest macroscopic length scale of the disk, i.e. half-thickness $h$, and the eddy velocity of turbulence $v_{t}$ in terms of sound speed $c_{s}$. Thus they defined the turbulent viscosity

$$
\nu_{t}=\frac{l_{t} v_{t}}{3}=\alpha c_{s} h
$$

where $l_{t}=\alpha_{l} h, v_{t}=\alpha_{v} c_{s}, \alpha=\alpha_{l} \alpha_{v} / 3$. Obviously $\alpha_{l} \leq 1$. If the turbulent velocity becomes supersonic, then shock forms and reduces the velocity below the sound velocity which assures $\alpha_{v} \leq 1$. Therefore, $\alpha \lesssim 1$. From eqns. (14) and (16) we write

$$
\bar{\alpha}=-\frac{T_{x y}}{q \Omega^{2}\left(\frac{h}{r}\right)^{3} M r^{2}},
$$

where $\bar{\alpha}$ denotes the averaged $\alpha$ in the small section. Therefore, if we know the structure of the flow, then we can compute the turbulent viscosity due to various perturbations. As we consider the size of the section to be very small, $\bar{\alpha}$ and $\bar{\nu}_{t}$ are effectively equivalent to $\alpha$ and $\nu_{t}$ at a particular position in the disk. Below we compute $T_{x y}$ for the various secondary perturbations and the corresponding turbulent viscosities, at least in certain approximations.

\subsection{Secondary perturbation evolves much rapidly than the primary one}

From eqn. (9) we can write the velocity perturbation components

$$
\begin{aligned}
& u_{x}(x, y)=A_{x} e^{\sigma t} f_{x}(\phi) \sin \left(k_{1} x+k_{2} y+k_{3} z\right), \\
& u_{y}(x, y)=A_{y} e^{\sigma t} f_{y}(\phi) \sin \left(k_{1} x+k_{2} y+k_{3} z\right),
\end{aligned}
$$

where $A_{x}$ and $A_{y}$ are the amplitudes of perturbation modes, $k_{10}, k_{20}$ are the radial and the azimuthal components respectively of the secondary perturbation wavevector at $t=0, A_{x}$ and $A_{y}$ can be evaluated by the condition that the velocity components of the secondary perturbation reduce to that of the primary perturbation at $t=0$ (at the beginning of the evolution of secondary perturbation) given by

$$
\begin{aligned}
A_{x} & =\zeta \frac{k_{y}}{l^{2}(\epsilon)} \frac{C}{f_{x}(0)}, A_{y}=-\zeta \frac{k_{x}(\epsilon)}{l^{2}(\epsilon)} \frac{C}{f_{y}(0)}, \\
C & =\frac{\sin \left(k_{x}(\epsilon) x+k_{y} y\right)}{\sin \left(k_{10} x+k_{20} y+k_{30} z\right)},
\end{aligned}
$$

where $k_{x}(\epsilon)=\sqrt{\epsilon /(1-\epsilon)} k_{y}, C$ is of the order of unity (for details see Mukhopadhyay et al. 2005. Mukhopadhyay 2006). Therefore, from eqn. (13)

$$
\begin{aligned}
T_{x y}\left(t_{g}\right) & \sim-\zeta^{2} \frac{k_{x}(\epsilon) k_{y}}{2 l^{4}(\epsilon)} e^{2 \sigma t_{g}} D, \\
D & =C^{2} \frac{f_{x}(\phi) f_{y}(\phi)}{f_{x}(0) f_{y}(0)} .
\end{aligned}
$$


Now by considering a typical case with $k_{y}=0.71, \nu_{t}$ and $\alpha$ can be computed as functions $\epsilon\left(k_{x}\right)$, when we know the time of evolution of the secondary perturbation $t_{g}$.

Figure 2 describes $\nu_{t}$ and $\alpha$ according to eqns. (14), (17) and (20) for various disk parameters. As the primary perturbation evolves, elliptical vortices form into the shearing flow which generate the turbulent viscosity under a further perturbation. Figure 2 a shows that the viscosity varies with the eccentricity of vortices. At a very early stage when the primary perturbation is effectively a radial wave and $\epsilon \rightarrow 1$, the maximum velocity growth rate due to secondary perturbation, $\sigma_{\max }$ (shown in Fig. 11), and the corresponding turbulent viscosity are very small, independent of the value of $\zeta$. With time, the primary perturbation wavefronts are straightened out by the shear until $t=t_{\max }$, when the perturbation becomes effectively an azimuthal wave and $\epsilon \rightarrow 0$. At this stage, $\sigma_{\max }$ and the turbulent viscosity due to the secondary perturbation become zero again. This feature is clearly understood from eqn. 20]. However, at an intermediate time when $k_{x}(\epsilon)$ is finite, $\nu_{t}$ may be $\sim 0.005$ even in a moderately slim disk with $h(r) / r=0.05$, when the time of evolution of secondary perturbation $t_{g}=10$. This $t_{g}$ is considered to be the time at which turbulence is triggered in the system. Figures $2 \mathrm{~b}$-d show the variation of $\nu_{t}$ and $\alpha$ with the eccentricity of vortices at various $\zeta$ when $t_{g}=10,100$. It is interesting to note, particularly for $t_{g}=100$, that with the increase of $\zeta$, first viscosity increases then decreases. This is understood from the underlying energy growth rate shown in Fig. 1, when the readers are reminded that $\sigma=\sigma(\zeta, \epsilon)$. Note that the qualitative behavior of $\nu_{t}$ is same as that of $\alpha$. If we look at a typical case with $\zeta=0.05$ where $\sigma=\sigma_{\max }$ at $\epsilon=0.86$ which corresponds to $k_{x}=-1.76$, then $\alpha$ and $\nu_{t}$ computed at $t=t_{g}$ are for $R e_{s} \lesssim R e_{p} \sim 10^{8}$.

\subsection{Secondary perturbation over the slowly varying primary perturbation}

In principle, the primary perturbation may vary with time during the evolution of secondary perturbation. By numerical solutions, simultaneous evolution of the primary and the secondary perturbation along with the corresponding energy growth has already been discussed earlier (Mukhopadhyay 2006). For the convenience of analytical computation of viscosity, here we consider the regime of slow variation of the primary perturbation compared to the secondary one. Hence we recall eqn. (10) and write the velocity perturbation components

$$
\begin{aligned}
& u_{x} \rightarrow u_{x_{\Sigma}}(x, y)=B_{x} e^{\Sigma(t)} f_{x}(\phi) \sin \left(k_{1} x+k_{2} y+k_{3} z\right), \\
& u_{y} \rightarrow u_{y_{\Sigma}}(x, y)=B_{y} e^{\Sigma(t)} f_{y}(\phi) \sin \left(k_{1} x+k_{2} y+k_{3} z\right),
\end{aligned}
$$

with $\phi=\int \varpi(t) d t$. The amplitudes of perturbation modes $B_{x}$ and $B_{y}$ can be evaluated by the initial condition of secondary perturbation. The secondary perturbation could trigger elliptical instability only after significant vortex forms in the flow due to the evolution of primary one. At the beginning of the evolution of primary perturbation $k_{x 0} \rightarrow-\infty$ (we choose the cases $k_{x 0}=-10^{5}$ and $-10^{4}$ ) which corresponds to $\epsilon \rightarrow 1$ and thus effectively a plane shear background when $\zeta$ is small (see Mukhopadhyay 2006). In absence of vortex, this can not trigger elliptical instability under a secondary perturbation. As $k_{x 0}$ decreases in magnitude, $\epsilon$ deviates from unity giving rise to a background consisting of elliptical vortices. Above certain $\epsilon=\epsilon_{c}$, the secondary perturbation does not have any effect to the primarily perturbed flow and $u_{x_{\Sigma}}$ and $u_{y_{\Sigma}}$ reduce to the primary perturbation. We hypothesize that $\epsilon_{c}=0.9999$. Hence, $B_{x}$ and $B_{y}$ are computed in a similar fashion as in $\S 3$.A given by

$$
\begin{aligned}
B_{x} & =\zeta \frac{k_{y}}{l^{2}\left(\epsilon_{c}\right)} \frac{C}{f_{x}(0)}, B_{y}=-\zeta \frac{k_{x}\left(\epsilon_{c}\right)}{l^{2}\left(\epsilon_{c}\right)} \frac{C}{f_{y}(0)}, \\
C & =\frac{\sin \left(k_{x}\left(\epsilon_{c}\right) x+k_{y} y\right)}{\sin \left(k_{10} x+k_{20} y+k_{30} z\right)} .
\end{aligned}
$$

Hence, from eqn. (13) the stress tensor

$$
\begin{aligned}
T_{x y}\left(t_{\max }\right) & \sim-\zeta^{2} \frac{k_{x}\left(\epsilon_{c}\right) k_{y}}{2 l^{4}\left(\epsilon_{c}\right)} e^{2 \Sigma_{\max }} D, \\
D & =C^{2} \frac{f_{x}(\phi) f_{y}(\phi)}{f_{x}(0) f_{y}(0)}
\end{aligned}
$$



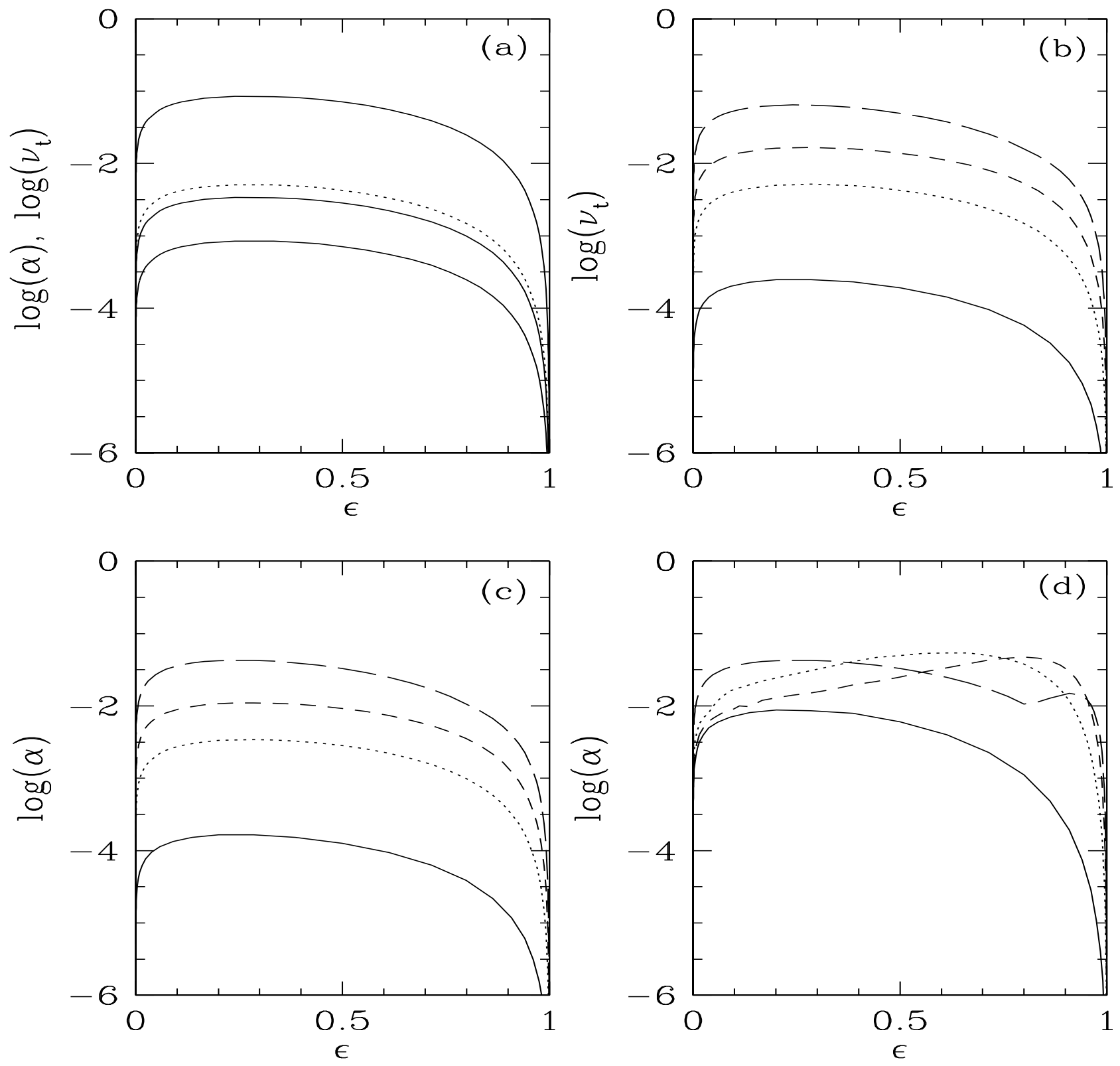

Fig. 2 This is for the perturbation described in $\S 3$.A. (a) Variation of $\nu_{t}$ (dotted curve) and $\alpha$ (solid curve) as functions of $\epsilon$ for $\zeta=0.05$ case described in Fig. 1 1 , when $h(r) / r=$ $0.01,0.05,0.1$ respectively for the top, middle, bottom curves of $\alpha ; r=30, k_{y}=0.71$, $t_{g}=10$. (b) Variation of $\nu_{t}$ as a function of $\epsilon$ for the cases described in Fig. 11 with $h(r) / r=0.05, t_{g}=10, k_{y}=0.71$, when solid, dotted, dashed, long-dashed curves correspond to $\zeta=0.01,0.05,0.1,0.2$ respectively with $\left|\boldsymbol{k}_{0}\right|=1$. (c) Same as in (b) except $\alpha$ is plotted in place of $\nu_{t}$. (d) Same as in (c) except $t_{g}=100$. 

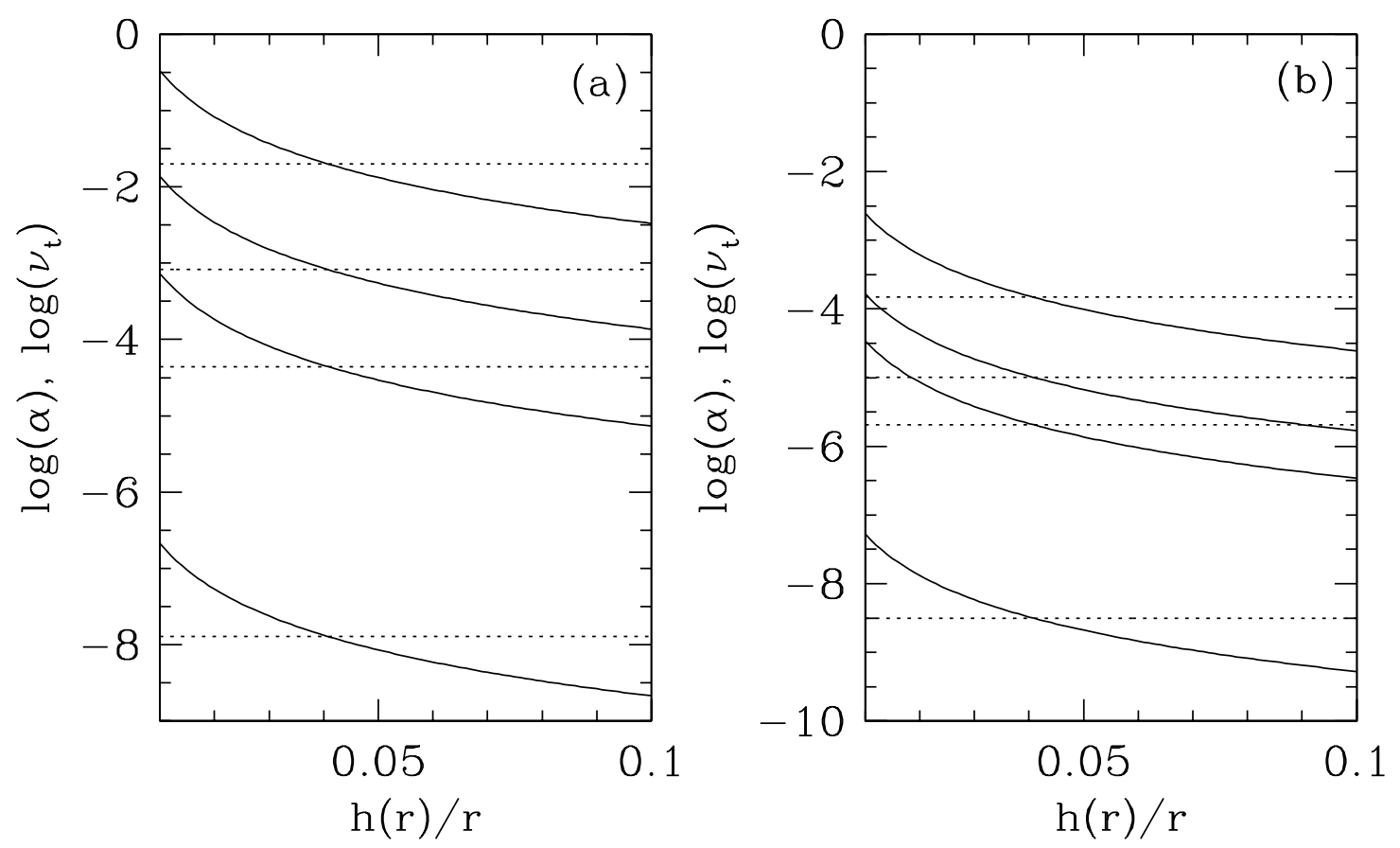

Fig. 3 This is for the perturbation described in $\S 3$.B. Variation of $\nu_{t}$ (dotted curve) and $\alpha$ (solid curve) as functions of $h(r) / r$ for cases shown in Figs. $1 \mathrm{~b}, \mathrm{c}$, when the curves from top to bottom correspond to $\zeta=0.2,0.1,0.05,0.01$ with $r=30$ for (a) $k_{x 0}=-10^{5}$, (b) $k_{x 0}=-10^{4}$. Other parameters are $k_{y}=1, \epsilon_{c}=0.9999$.

where $k_{x}$ reduces to zero at $t=t_{\max }$, which corresponds to the beginning of turbulence when $\Sigma=\Sigma_{\max }$.

It is found from Fig. 3 that in a thin disk with $h(r) / r=0.01, \alpha$ at $r=30$ may be as high as $\gtrsim 0.1$ for $k_{x 0}=-10^{5}$ when $\zeta$ is very large. Although the viscosity decreases with the decrease of $\zeta, \alpha$ still may be $\sim 0.001$ when $\zeta=0.05$. The turbulent viscosity decreases in a considerably thicker disk, but still 
$\alpha \sim 0.003$ at $h(r) / r=0.1$ when $\zeta=0.2$. For $\zeta \geq 0.1, \nu_{t} \geq 0.001$ when $k_{x 0}=-10^{5}$. The values of $\nu_{t}$ and $\alpha$ both decrease when $\left|k_{x 0}\right|$ decreases to $10^{4}$, which is expected from Table 1 as well. In this case, a significant turbulent viscosity generates only at a large $\zeta=0.2$.

\section{IMPLICATIONS AND DISCUSSIONS}

Above results verify that at a range of $\epsilon$, the threedimensional growth rate due to secondary perturbation in rotating shear flow of the Keplerian kind is always real and positive and corresponding growth may be exponential and significant enough, at least for a suitable choice of $\zeta$ and/or $R e$, to trigger non-linearity and then plausible turbulence in the flow time scale. With the increase of $k_{x 0}\left(\sim R e_{p}{ }^{1 / 3}\right)$, the effect due to elliptical instability increases, and thus corresponding growth does so.

As this growth is the result of threedimensional perturbation, underlying perturbation effect should survive even in the presence of viscosity. There are many important natural phenomena where the Reynolds number is very large. In astrophysical accretion disks, what applications are essentially considered in the present paper, $R e$ always could be $\gtrsim 10^{10}$ because of their very low molecular viscosity. Therefore, the present mechanism is certainly applicable to such disk flows to resolve their turbulence puzzle when especially it is cold and neutral in charge and thus not a very plausible candidate for the magnetorotational instability. On the other hand, we suggest that the subcritical transition to turbulence in Couette flow may be the result of secondary perturbation which triggers elliptical instability modes into the system.

We have tried to estimate the corresponding hydrodynamic turbulent viscosity. We have aimed to quantify the amount of turbulence through this using the perturbations as the source of turbulence. We report here an observable range of viscosity obtained for the typical thin accretion disks and with reasonable values of flow vorticity. In place of $r=30$, if we choose the shearing box at a large distance from the central object, say at $r=500$, then the computed $\alpha$ naturally decreases three orders of magnitude [see eqn. (17)]. We show by an extensive analysis the dependence of viscosity on the aspect ratio $(h / r)$ of the flow. The values of $\nu_{t}$ and $\alpha$ increase quite rapidly as the disk becomes thin to thinner. From eqns. (14) and (17) and with the results given in Figs. 2 and 3, we find that it still might be as large as $10^{-4}$ for a thin disk even at a large distance, say, $r=500$.

While some earlier laboratory experiments (e.g. Richard \& Zahn 2001) predicted sub-critical transition to turbulence and then transport in hydrodynamical shear flows like accretion disks, experiments by Ji et al. (2006) have argued against it. Non-detection of turbulence and then any angular momentum transport of purely hydrodynamic origin could be due to the following facts. Maximum Reynolds number in this experiment is $2 \times 10^{6}$ whereas the cold disks such as the protoplanetary disks have Reynolds number $\sim 10^{12}$. However, the critical Reynolds number for these systems could be $\sim 10^{6}-10^{7}$ or more. It can be easily understood with a very simple example that as $R e$ increases, the amplitude of vortices increases which are indeed clear from the Figs. 7 and 8 given by Mukhopadhyay et al. (2005). Let us consider a 2D perturbation in an inviscid incompressible flow where the vorticity $\nabla \times \boldsymbol{v}$ is exactly conserved, when $\boldsymbol{v}=\hat{i} v_{x}+\hat{j} v_{y}$. Therefore, at $t=t_{\max }=t_{g}$, when the perturbation growth is maximum at $t=t_{\max }$, the amplitude of vorticity $\zeta \sim|l v| \sim R e^{1 / 3}$. As $\nu_{t}$ and $\alpha$ are directly proportional to $\zeta^{2}$, they scale as $R e^{2 / 3}$ at $t=t_{\max }=t_{g}$. Therefore, if $R e$ decreases three orders of magnitude, then $\nu_{t}$ decreases in two orders. Moreover, the perturbation stabilizes at a thicker disk. Indeed we find that the viscosity decreases, as $h(r) / r$ increases. Dimension of confined liquid in the experiments by Ji et al. (2006) may not be typical of astrophysical disks or rings, when they may have a large aspect ratio $\sim 2$, whereas the astrophysical disks and ring systems are normally thin (with aspect ratio $\leq 1$ ). Obviously a huge gap exists between experiments and the real observations.

By numerical simulations, the formation and evolution of vortices in a hydrodynamic shearing-sheet have already been studied by Johnson \& Gammie (2005) and they suggested it to be a possible mechanism for angular momentum transport in low-ionization disks at high resolution. It has been argued that there must be a mechanism to inject vorticities into the disk, and the vortices must not decay rapidly due to threedimensional instabilities, to sustain the transport. We show that the vortices may sustain in threedimension at least in the time scale of interest, where this is applicable for accretion disks. Indeed, Cuzzi and his collaborators (Cuzzi 2007, Ormel et al. 2008) have argued, by numerical simulations, that the elliptical instability may lead to turbulence to from the dusty gas surrounding a young star. Also the vortex generation and 
then the angular momentum transport has been shown to occur in the unmagnetized protoplanetary disks (de Val-Borro et al. 2007) by hydrodynamic turbulence. However, other simulations (Shen et al. 2006) do not find significant transport. The nonoccurence of significant transport in simulations, in our view, is due to lack of resolution needed to capture the turbulence. Indeed, the later authors have mentioned that for their calculations it is difficult to define an effective Reynolds number, since the numerical dissipation is a steep function of resolution. With a particular non-linear solution, Balbus \& Hawley (Balbus \& Hawley 2006) have shown that perturbation decays asymptotically. They also have argued that as the nonlinear term in the equation for the incompressible flow itself vanishes explicitly, the solution can not lead to nonlinearity and then turbulence. However, this does not guaranty that every solution does so. They themselves have also mentioned that secondary instabilities may still spoil their conclusion. Indeed the coupling between the secondary and primary modes was shown earlier not to allow the nonlinear term to vanish resulting in a possible nonlinear transition to turbulence (Mukhopadhyay 2006).

It is interesting to note that the modal instability via the bypass mechanism (and then with a secondary perturbation superimposed) arises in these systems from a subtle interplay of the non-normality of the perturbation modes and the non-linearity of the Navier-Stokes equation and this in turn gives rise to the turbulence in the system. As the turbulence and corresponding transport is inevitable in these systems, the corresponding $\alpha$ may not be just inversely proportional to the critical Reynolds number (as predicted earlier (Lesur \& Longaretti 2005)). Previous theoretical studies (Mukhopadhyay et al. 2005) have shown that the Keplerian flow may render a transition to the turbulent regime at a Reynolds number $\sim 10^{6}$ and turbulence might have just started at this critical Reynolds number. It is to be seen now whether all shear flows, exhibiting subcritical turbulence in the laboratory, do exhibit large growth due to secondary perturbation.

Acknowledgements This work is partly supported by a project, Grant No. SR/S2HEP12/2007, funded by DST, India.

\section{References}

S. A. Balbus, \& J. F. Hawley, ApJ 652, 1020 (2006).

B. Bayly, Phys. Rev. Lett. 57, 2160 (1986).

K. Bech, \& H. Andersson, J. Fluid Mech. 347, 289 (1997).

O. Blaes, \& S. Balbus, ApJ 421, 163 (1994).

K. Butler, \& B. Farrell, Phys. Fluids A 4(8), 1637 (1992).

G. Chagelishvili, J.-P. Zahn, A. Tevzadze, \& J. Lominadze, A\&A 402, 401 (2003).

A. Craik, J. Fluid Mech. 198, 275 (1989).

A. Craik, \& W. Criminale, Proc. R. Soc. London Ser. A 406, 13 (1986).

W. O. Criminale, T. L. Jackson, \& R. D. Joslin, in Theory and Computation in Hydrodynamic Stability (Cambridge:

Cambridge Univ. Press) (2003).

J. N. Cuzzi, Nature 448, 1003 (2007).

M. de Val-Borro, P. Artymowicz, G. D’Angelo, \& A. Peplinski, A\&A 471, 1043 (2007).

C. Gammie, \& K. Menou, ApJ 492, L75 (1998); K. Menou, Science 288 (5473), 2022 (2000).

C. Hellberg, \& S. Orszag, Phys. Fluids 31(1), 6 (1988).

H. Ji, M. J. Burin, E. Schartman, \& J. Goodman, Nature 444, 343 (2006).

B. M. Johnson, \& C. F. Gammie, ApJ 635, 149 (2005)

R. Kerswell, Ann. Rev. Fluid Mech. 34, 83 (2002).

M. Landman, \& P. Saffman, Phys. Fluids 30(8), 2339 (1987).

S. Le Diześ, M. Rossi, \& K. Moffatt, Phys. Fluids 8(8), 2084 (1996).

G. Lesur, \& P.-Y. Longaretti, A\&A 444, 25 (2005).

K. Menou, \& E. Quataert, ApJ 552, 204 (2001).

B. Mukhopadhyay, ApJ 653, 503 (2006).

B. Mukhopadhyay, IJMPD 17467 (2008). 
B. Mukhopadhyay, N. Afshordi, \& R. Narayan, ApJ 629, 383 (2005); N. Afshordi, B. Mukhopadhyay, \& R. Narayan, ApJ 629, 373 (2005).

C. W. Ormel, J. N. Cuzzi, \& A. G. G. M. Tielens, ApJ 679, 1588 (2008).

R. Pierrehumbert, Phys. Rev. Lett. 57, 2157 (1986).

S. Reddy, \& D. Henningson, J. Fluids Mech. 252, 209 (1993).

D. Richard, \& J.-P. Zahn, A\&A 347, 734 (1999).

P. J. Schmid, \& D. S. Henningson, in Stability and Transition in Shear Flows (New York: Springer-Verlag) (2001).

N. I. Shakura, \& R. A. Syunyaev, A\&A 24, 337 (1973).

Y. Shen, J. M. Stone, \& T. A. Gardiner, ApJ 653, 513 (2006).

L. Trefethen, A. Trefethen, S. Reddy, \& T. Driscoll, Science 261, 578 (1993).

O. Umurhan, \& O. Regev, A\&A 427, 855 (2004).

F. Waleffe, Phys. Fluids A 2(1), 76 (1989).

This was prepared with the RAA LATEX macro v1.0. 